\title{
Behavioral Interventions in Autism
}

\author{
Vijaya Lakshmi Chouhan ${ }^{1}$, Preeti Sharma ${ }^{2} *$
}

\section{ABSTRACT}

Childhood psychiatric disorders have increased over the last decades. Changing socio-cultural environmental conditions, better diagnostic techniques and increased sensitization among the masses and the health professionals are some of the most common reasons of this increased childhood psychiatric diagnoses. Autism spectrum disorders (ASD) are one of the most common disorders among children. it is a group of neurodevelopment disorders with specific delays and deviance in social, communicative and cognitive development. It includes autism, Asperger's syndrome, and pervasive developmental disorder- not otherwise specified (PDD-NOS). It is a lifelong developmental condition that affects the way an individual relates to his or her environment and their interaction with other people. In this paper, the term ASD and autism will be used interchangeably. It is often diagnosed between the age of two and five years. biopsychosocial model is used to explain the possible sources of origin of autism spectrum disorder. Medical, cognitive, behavioral, early intervention approaches are the most common models of interventions in autism. The current paper will specifically describe the various behavioral interventions commonly used in the management of autism.

Keywords: Autism, PDD, behavioral interventions, ASD

Autism and autistic spectrum disorders (ASDs) are life long neurodevelopment disorders affecting sociability and communication for which no etiology-based treatment has yet been developed. The word "spectrum" describes the range of difficulties that autistic people may experience and the degree to which they may be affected. The term autism spectrum disorders (ASDs) has been used to include the Diagnostic Statistical Manual of Mental Disorders, Fourth Edition, Text Revision (DSM-IV-TR, 2000) diagnostic categories autistic disorder, Asperger disorder and pervasive developmental disorder- not otherwise specified. The main areas of difficulty are in social interaction, verbal and nonverbal communication and restricted or repetitive behaviors and interests. The word "autism" comes from the greek word "autos",

\footnotetext{
${ }^{1}$ President, Academy of Well-being \& Former Dean, Students’ Welfare Board, MLSU,HOD, Department of Psychology, Udaipur, Rajasthan, India

${ }^{2}$ Counselor, Academy of Well-being, Udaipur \& Research Scholar, Pacific University, Udaipur, India

*Responding Author
}

Received: December 20, 2016; Revision Received: January 12, 2017; Accepted: January 27, 2017

(C) 2017 Chouhan V, Sharma p; licensee IJIP. This is an Open Access Research distributed under the terms of the Creative Commons Attribution License (creativecommons.org/licenses/by/2.0), which permits unrestricted use, distribution, and reproduction in any Medium, provided the original work is properly cited. 


\section{Behavioral Interventions in Autism}

meaning "self." the term describes conditions in which a person is removed from social interaction meaning an "isolated self". Children with autism find it difficult to act in a way that other people think is "normal". The term autism was coined by Bleuler in 1911. Freud equated autism with narcissism. In the beginning, autism was known as childhood schizophrenia. Leo Kanner described it in 1943. He found some children who found it difficult to relate to others, a failure to use language to convey meaning and an inordinate desire for the maintenance of the same.

ASDs are in the range of 6.5 to 6.6 per 1000 globally. Malhotra, Chakrabarti, Gupta, Kumar, and Gill (2003) estimate that out of 2942 cases registered at child and adolescent psychiatry clinic at PGIMER, Chandigarh, 1.6\% met ICD 10 criteria of PDD. Out of these cases, 22 cases belong to ASD category. Bharat, Srinath, Sheshadri, and Girimaji (1997) reviewed in-patient data for one year data at child and adolescent psychiatry clinic NIMHANS, Bangalore and found that out of 143 registered cases, 6 belong to ASD.

As specified in DSM-IV-TR (American Psychiatric Association, 2000), autism spectrum disorders involve limitations in social relatedness, verbal and non-verbal communication and range of interests and behaviors. In the social domain, symptoms include impaired use of nonverbal behaviors (e.g., eye contact, facial expressions, gestures) to regulate social interactions, failure to develop age appropriate peer relationships, little seeking to share enjoyments and interests with other people and limited socio-emotional reciprocity. Communication deficits include delay in development of or absence of spoken language, difficulty in initiation and maintaining conversation; idiosyncratic or repetitive language and imitation pretend play deficits. In the behaviors and interests domain, there are often encompassing, unusual interests, inflexible adherence to non-functional routines, stereotypes body movement like hand flapping, flick fingers in front of eyes, tip toe walking and preoccupation with parts or sensory modalities of objects (American Psychiatric Association, 2000). Children with autism find it difficult to talk to other people and to look at other people. Often, they do not like being touched by other people. A person who has autism seems to be turned inwards. They may talk only to themselves, rock themselves backwards and forwards, and laugh at their own thoughts. They do not like any type of change and may find it very difficult to learn a new behavior like using a toilet or going to school.

To meet the criteria for autistic disorder, an individual must demonstrate 6 of the 12 symptoms, with at least 2 coming from the social domain and 1 each from the communication and restricted behaviors/interests categories. At least 1 symptom must have been present before 3 years. The onset of autism occurs before age 3, at two peak periods. The majority of children display developmental abnormalities within the first 2 years of life. Most individuals with autism improve with time and age. Symptoms particularly the repetitive and stereotypic behaviors 


\section{Behavioral Interventions in Autism}

appear to increase for a few years after onset, usually peaking in the preschool period, but begin to level off or decline in the school-age years.

The ability of central coherence is weak in children with autism. It is the ability to build wholes out of parts. This means that people with autism focus more on details. They cannot build wholes out of them. Not being able to pay attention to wholes results in difficulties with perception and language. Therefore, children with autism usually need more time for dealing with information coming from their surroundings. As a consequence, they typically also need more time for responding. Impairment in central coherence is known as simultanagnosia. However, performance varies between people on the autism spectrum.

During the last few decades, however, the knowledge concerning the associated deficits of the disorder has rapidly expanded and many psychological, educational and medical interventions have been proposed, claiming to be effective. Children on the autism spectrum have impairment in executive function. Executive function refers to physical, emotional, and cognitive selfcontrol. This includes planning actions, focusing, shifting attention, flexibility of behaviour and thinking and deficits in working memory. A smaller corpus callosum leads to abnormalities in working memory (an active process of keeping a memory until it is needed) and planning actions (Keary, Minshew, Bansal, Goradia, Fedorov, Keshavan, et al. (2009). Pisula (2010) argues that problems with executive function are probably caused by large networks in the brain. O'hearn, Asato, Ordaz., and Luna (2008) assert that grey matter and white matter irregularities have a negative influence on the way different brain regions work together (functional integration).

\section{Autism and its co-morbidity}

Autism can co-occur with a variety of other neurocognitive developmental disorders. Best appreciated is the high comorbidity rate of autism and mental retardation. Most studies have found that the majority of individuals with autism (75\%) are intellectually handicapped, with roughly half of the group functioning in the range of mild to moderate mental retardation and half in the severe to profound range.

Another commonly associated condition with autism is seizures. Some children with autism suffer from seizures, with onset most often occurring during either the pre-school or adolescent years. Autism also seem to co-occur with Tourette syndrome () and other tic disorders. Attention Deficit Hyperactivity Disorder (ADHD) is yet another common co-morbid condition with autism.

Autism has been found to be more common among males than females with a ratio of 4:1.It is now accepted that autism occurs all socioeconomic levels, in all cultures and in all ethnic and racial groups. 


\section{Behavioral Interventions in Autism}

Genetic and psychological factors (Theory of Mind) both play an etiological role in the development of autism.

\section{Psychological management of Autism}

Before deciding the interventional strategies in the management of autism, it is always important to do through psychological assessment of a child. The psychological assessment of a child includes

- Level of intellectual ability

- $\quad$ Adaptive functioning specifically in the domain of self-help and social skills

- $\quad$ Specific assessment using autism scales and

- Behavioral problems

Autism like other neurocognitive developmental disorders is generally "not curable" and chronic management is required. The primary goals of treatment are to minimize the core features and associated deficits, maximize functional independence and quality of life and alleviate family distress. Facilitating development and learning, promoting socialization, reducing problem behaviors and psycho educating and supporting families can help accomplish these goals. Ideally, interventions should help mitigate the core features of autism like impairment in social reciprocity, deficits in communication, and restricted, repetitive behavioral repertoire. Behavioral strategies are the cornerstones and are most important and widely used in the management of autism. These interventions address

$\begin{array}{ll}\text { - } & \text { social skills } \\ \text { - } & \text { problem behaviors, } \\ \text { - } & \text { daily-living skills, } \\ \text { - } & \text { communication } \\ \text { - } & \text { play and leisure skills }\end{array}$

The whole objective is to make child's functionally independent to the extent possible and teach life skills.

\section{Behavioral interventions:}

Behavioral interventions refer to those interventions where instrumental/Skinnerian based techniques form the predominant feature of the approach. Contemporary comprehensive behavioral curricula borrow from developmental or cognitive approaches such as addressing joint-attention, reciprocal imitation, symbolic play, perspective taking, self-management, social initiation, decreases problem behaviors and theory of mind and using indirect language stimulation and contingent imitation techniques and some developmental models e.g. Denver model and the structured teaching approach of the Treatment and Education of Autistic and Related Communication Handicapped Children (TEACCH, Schopler, 1984) program uses behavioral techniques to fulfill their curriculum goals. Behavioral techniques of reinforcement (mainly positive), backward chaining (i.e. the process of teaching each component of a behavior 


\section{Behavioral Interventions in Autism}

starting with the last step needed to complete the sequence), shaping, and prompt and prompt fading are used. Physical punishment is no longer used. Initially, food and favorite objects are used as reinforcers and are later replaced by more social ones such as praise. Learned responses are repeated until firmly embedded. Applied Behavioral Analysis (ABA) and discrete Trial Training have been the latest techniques introduced in the domain of behavioral treatment. Two or three hour sessions. short periods of structured time devoted to a task (3-5 minutes) followed by an equal amount of free play for the child (3-5 minutes). Longer break (10-15 minutes) at the end of every hour. Free play and breaks are used for incidental teaching or practicing learned skills in new environments. 35-40 hours per week. Breaking down skills into manageable pieces and then builds upon those skills so that a child learns how to learn in the natural environment.

Although programs may differ in philosophy and relative emphasis on particular strategies, they share many common goals and there is a growing consensus that important principles and components of effective early childhood intervention for children with autism include the following:

- entry into intervention as soon as autism diagnosis is seriously considered;

- provision of intensive intervention with active engagement of the child at least 25 hours per week, 12 months per year, in systematically planned, developmentally appropriate educational activities designed to address identified objectives;

- low child-to-professional ratio to allow sufficient amounts of 1-on-1 time and small-group instruction to meet specific individualized goals;

- incorporation of a high degree of structure through elements such as predictable routines, visual activity schedules and clear physical boundaries to minimize distractions;

- implementation of strategies to apply learned skills to new environ -ments and situations ( generalization) and to maintain functional use of these skills;

\section{Specific Strategies}

A variety of specific interventional techniques are used in psychological management programs for children with autism. These are aimed to teach social skills, reduce problem behaviors and enhance communication. These techniques are as follow:

\section{Applied Behavior Analysis (ABA)}

In the ABA approach the focus is on the use of rewards or reinforcements to encourage desirable behaviors and the reduction of problem behaviors by their positive consequences by means of different techniques like "time-out" "extinction" or withholding of child's privileges following the undesirable problem. This was first used by an American psychologist Lovass in 1989 with autistic children to improve their social interaction and decrease maladaptive behaviors. A detail initial assessment is required to determine the level of functioning and specific behavioral difficulties. Careful observation is needed to identify the triggers of problem behavior and events that appear to maintain or encourage either positive or negative behaviors. 


\section{Behavioral Interventions in Autism}

$\mathrm{ABA}$ focuses on the reliable measurement and objective evaluation of observable behavior within relevant settings including the home, school, clinics and community.

Functional behavioral analysis or functional assessment is an important aspect of behaviorally based treatment of unwanted behaviors. Most problem behaviors serve as an adaptive function of some type and are reinforced by their consequences such as attainment of parent's attention, a desired object, activity or sensation or escape from undesirable situation or demand. Functional assessment is a rigorous, empirically based method of gathering information that can be used to maximize the effectiveness and efficacy of behavioral interventions.

It uses $\mathrm{ABC}$ model where "A" stands for antecedent conditions that initiate or trigger problem behavior like any situation, place, cues etc while "B" is the actual behavior displayed by the child like hitting, crying, banging the head, and finally " $\mathrm{C}$ " stands for the consequences following the child's behavior like parent's attention, cuddling the child etc.It is theorized that consequences following the child's behavior (B) is responsible either to maintain or decrease it. Following the $\mathrm{ABC}$ analysis, the frequency and intensity of the problem behaviors, their triggering and maintaining factors are identified. The parents are then primarily trained to modify their responses subsequent to the child's problem behavior if it is found that parent's behaviors are responsible for maintaing problem behaviors. Parents are also trained to give positive attention to desirable behaviors like child sitting quietly and trying to initiate in play activities so that these behaviors are strengthened.

The goal of ABA technique is to teach child

- Desirable behaviors

- Decreases undesirable behaviors

- Improve social and other new skills

- Imitation skills

- Preacademic skills and

- Self-help skills

\section{Discrete Trial Training (DTT)}

As a part of broader ABA intervention, discrete trials can be used to target numerous goals and objectives that children need to be explicitly taught. New skills are taught in a graduated step-bystep manner called discrete trial teaching. It is a behavioral and educational strategy based on the principles of ABA involving breaking skills down into smaller components and teaching those smaller sub-skills individually. Instrunctions are very clear, concise and repetitive. Repeated practice of the skills is conducted and professionals may incorporate prompting procedures. Correct responses are followed by reinforcement to facilitate learning. The basic rationale of discrete trial training is that children with autism typically do not learn from their surroundings spontaneously and hence often need to be explicitly taught virtually every thing they are expected to learn (Green, 1995). A discrete trial consists of 5 main parts for e.g.

1. An initial instruction e.g. "Nose your eyes." 


\section{Behavioral Interventions in Autism}

2. A prompt or cue given to help the child respond correctly e.g. professional points to the child's eyes.

3. A response given by the child e.g. a child touches his/her eyes.

4. A correct response by the child leads to getting reward like smiley stickers; verbal reward “ nice job” designed to motivate the child to respond correctly again.

5. A pause between trials waiting 1-5 seconds before beginning the next trial.

In discrete trial training, simple skills are mastered before new learning opportunities are presented in which the child then builds upon the mastered skill toward a more complex one. Discrete trial training are used in the development of early skills like

- $\quad$ proper sitting (sit down, head quiet)

- directing attention

- $\quad$ compliance/following verbal instructions

- $\quad$ imitation of simple actions ( gross motor, facial expressions, gestures)

- verbal imitation

- $\quad$ visual stimuli matching

- discrimination learning

There is a significant body of validated and peer-reviewed studies supporting the efficacy of DTT as a method to teach skills and improve and sustain socially significant behaviors in children with autism. Importantly, results reported include meaningful outcomes such as increased social and communication skills, self-help skills, functional skills, cognitive functions and academic performance. DTT has been criticized because of problems with generalizations of learned behaviors to spontaneous use in natural environments and because the highly structured teaching environment is not representative of natural adult-child interactions. Traditional ABA techniques have been modified to address these issues. It is to be remembered, however, that discrete trial training should be combined with other interventions to allow children to use skills in other settings and situations. Naturalistic behavioral interventions such as loosely structured training, incidental training, free operant structured training may enhance generalization of skills. These other behavioral methods based on ABA techniques are

1. Loosely Structured Training: It is used for situations when children with autism do not need the tightly controlled learning situations provided in DTT. In such training programs professionals select teaching material and tasks but follow a more flexible format than in DTT. For example, they set up a schedule presented in a pictorial format for children with autism to follow rather than giving instructions at each step. Also they may use modeling technique for the child to copy rather than demonstrating the skills themselves. This also involves behavioral skills training (BST) in which the professional/therapist gives an instructions, model an appropriate response, have individual rehearse this response and give 


\section{Behavioral Interventions in Autism}

feedback and reinforcement for the response. This can be used with a small group of children.

2. Incidental Teaching Training: In this, the professional/therapist sets up the environment that encourages the child to initiate the activities and instruction is given to her/him in the context of the activities the child has chosen. For e.g, the therapist may put toys in sight but out of reach and whenever the child attempts to gain access to one of the toys, the child is asked, "what do you want"? and require that the child name the toy in order to obtain access to it. It is assumed to improve communication and socio-cognitive skills.

3. Free Operant Instruction Training: In this training approach the therapist reinforce appropriate behavior and discourages problem behavior whey occur, but environment is not systematically arranged or cues are not provided to the child. For e.g. if the therapist may aim to " catch children being good" (e.g., praise children when they are playing quietly and appropriately with toys).

The effectiveness of ABA based interventions in the management of autism has been well documented through 5 decades of research by using single subject- methodology and in control studies of comprehensive early intensive behavioral interventions programs in structured and community settings. Children who receive early intensive behavioral treatment have been shown to make substantial, sustained gains in IQ, adaptive behavior, measures of social behavior, language and academic performance and yields a high degree of parental satisfaction and their outcomes have been significantly better than those of children in control groups.

\section{CONCLUSION}

Based upon the current findings, the most effective elements for an intervention are behavioral techniques and structured teaching based on visual cues. Multidisciplinary approach is the best line of intervention and finally, it is recommended that a comprehensive approach is favored with individualized treatment goals and programs involving parents and other professionals working with children with autism in a reciprocated manner.

\section{Acknowledgments}

The Author Appreciates All Those Who Participated In The Study And Helped To Facilitate The Research Process.

\section{Conflict Of Interests}

The Author Declared No Conflict Of Interests.

\section{REFERENCES}

Bharat, S., Srinath, S., Sheshadri, S.P., \& Girimaji, S. (1997). Child and Adolescent Psychiatry in Patient Facility. Indian Journal Psychiatry, 64(6), 829-832. 


\section{Behavioral Interventions in Autism}

Diagnostic and Statistical Manual Of Mental Disorders, 4th Edition, Text Revision. (2000). Washington Dc:, American Psychiatric Association.

Kanner, L. (1943). Autistic Disturbance Of Affective Content. Nervous Child, 2, 217-250.

Keary, C. J., Minshew, N. J., Bansal, R., Goradia, D., Fedorov, S., Keshavan, M. S., Et Al. (2009). Corpus Callosum Volume And Neurocognition In Autism. Journal Of Autism And Developmental Disorders, 39(6), 834-841.

Lovaas, O.I. \& Smith, T. (1989). A Comprehensive Behavioral Theory Of Autistic Children: Paradigm For Research And Treatment. Journal Of Behavior Therapy And Experimental Psychiatry, 20, 17-29.

Malhotra, S., Chakrabarti, S., Gupta, N., Kumar, P., \& Gill, S. (2003). Pervasive Developmental Disorders And Its Subtypes: Sociodemographic And Clinical Profile. German Journal Of Psychiatry, 4, 51-55.

O'hearn, K., Asato, M., Ordaz, S., \& Luna, B. (2008). Neurodevelopment And Executive Function In Autism. Development And Psychopathology, 20(04), 1103-1132

Pisula, E. (2010).The Autistic Mind In The Light Of Neuropsychological Studies. Acta Neurobiologiae Experimentalis, 70(2), 119-130

Schopler, E., Mesibov, G.B., \& Bashward, A. (1984). Helping Autistic Children Through Their Parent: The Teach Model. In Schopler \& Mesibov, (Eds.). The Effects Of Autism On The Family, Pp 65-81. New York: Plenum Press.

How to cite this article: Chouhan V, Sharma P (2017), Behavioral Interventions in Autism, International Journal of Indian Psychology, Volume 4, Issue 2, No. 85, ISSN:2348-5396 (E), ISSN:2349-3429 (P), DIP:18.01.010/20170402, ISBN: 978-1-365-68608-5 